\title{
Time in therapeutic range, quality of life and treatment satisfaction of patients on long-term warfarin for non-valvular atrial fibrillation: a cross-sectional study
}

Diana-Leh-Ching Ng${ }^{1}$, Natasya Marliana Bt Abdul Malik², Chee-Shee Chai', Greta-Miranda-Kim-Choo Goh², Seng-Beng Tan ${ }^{3}$, Ping-Chong Bee ${ }^{3}$, Gin-Gin Gan ${ }^{3}$ and Asri B. Said ${ }^{*}$ (D)

\begin{abstract}
Background: The use of warfarin in patients with non-valvular atrial fibrillation (NVAF) can be challenging. In this study, we evaluate the time in therapeutic range (TTR), health-related quality of life (HRQoL) and treatment satisfaction of patients on long-term warfarin for NVAF. The HRQoL and treatment satisfaction were compared based on the TTR.

Methods: A cross-sectional study was conducted among patients on warfarin for NVAF who attended the anticoagulant clinic of a tertiary cardiology referral center in Sarawak from 1st June 2018 to 31st May 2019. Patients'TTR was calculated by using Rosendaal technique, while their HRQoL and treatment satisfaction were assessed by using Short Form 12 Health Survey version 2 (SF12v2) and Duke Anticoagulant Satisfaction Scale (DASS), respectively.

Results: A total of 300 patients were included, with mean TTR score of $47.0 \pm 17.3 \%$. The physical component summary (PCS) and mental component summary (MCS) score of SF-12v2 were $47.0 \pm 9.0$ and $53.5 \pm 9.6$, respectively. The total score for DASS was 55.2 \pm 21.3 , while the score for limitations (L), hassles and burdens (H\&B) and positive psychological impacts (PPI) were $18.0 \pm 10.0,15.6 \pm 9.1$ and 21.6 \pm 5.9 , respectively. Seventy-three (24.3\%) patients had good TTR ( $\geq 60 \%$ ), with mean of 70.2 $\pm 8.7 \%$; while 227 (75.5\%) patients with poor TTR had significantly lower mean of $39.5 \pm 11.9 \%(p=0.006)$. There was no significant difference in the score of PCS $(p=0.150)$, MCS $(p=0.919)$ and each domain of SF-12V2 ( $p=0.184-0.684)$ between good and poor TTR, except for social functioning $(p=0.019)$. The total DASS score was also not significantly different between group $(p=0.779)$. Similar non-significant difference was also reported in all the DASS sub dimensions ( $p=0.502-0.699)$.

Conclusions: Majority of the patients on long-term warfarin for NVAF in the current study have poor TTR. Their HRQOL and treatment satisfaction are independent of their TTR. Achieving a good TTR do not compromise the HRQoL and treatment satisfaction. Therefore, appropriate measures should be taken to optimise INR control, failing which direct oral anticoagulant therapy should be considered.
\end{abstract}

Keywords: Warfarin, Time in therapeutic range, Quality of life, Treatment satisfaction, Direct oral anticoagulant

*Correspondence: sasri@unimas.my

1 Department of Medicine, Faculty of Medicine and Health Science, University Malaysia Sarawak, Kota Samarahan, Sarawak, Malaysia

Full list of author information is available at the end of the article

\section{Background}

Atrial fibrillation (AF) is the commonest arrhythmia and represents substantial health care burden globally [1]. In 2010, the estimated prevalence of AF was 592 per 100,000 original author(s) and the source, provide a link to the Creative Commons licence, and indicate if changes were made. The images or other third party material in this article are included in the article's Creative Commons licence, unless indicated otherwise in a credit line to the material. If material is not included in the article's Creative Commons licence and your intended use is not permitted by statutory regulation or exceeds the permitted use, you will need to obtain permission directly from the copyright holder. To view a copy of this licence, visit http://creativecommons.org/licenses/by/4.0/. The Creative Commons Public Domain Dedication waiver (http://creativeco mmons.org/publicdomain/zero/1.0/) applies to the data made available in this article, unless otherwise stated in a credit line to the data. 
in men and 360 per 100,000 in woman [2]. The risk of developing AF doubles with each progressive decade and exceeds $20 \%$ by 80 years old [3]. AF is associated with a five-fold increase in thromboembolic events, particularly stroke [4]. Studies have shown that one in six embolic strokes are attributed to underlying AF [5]. Without anticoagulant, the risk of embolic stroke in patients with AF ranges between 1.9 and $18.2 \%$ [6]. The common causes of AF include hypertension, diabetes mellitus, obesity, congestive heart failure, coronary heart disease, rheumatic heart disease and valvular heart disease [7]. Non-valvular $\mathrm{AF}$ (NVAF) is defined as AF not due to mitral disease or metallic valves [8].

Warfarin is a vitamin $\mathrm{K}$ antagonist broadly used to prevent embolic stroke in patients with AF. The therapeutic efficacy of warfarin is measured in international normalised ratio (INR). The target range of INR varies depending on the indications for anticoagulation [9]. The INR range recommended by American College of Chest Physician for patients with NVAF is $2.0-3.0$ [9]. The time in therapeutic range (TTR) is defined as the percentage of time the patients INR was within the target range. Patients with higher TTR value have been reported to have better outcome such as lesser stroke, major haemorrhagic events and death [10].

The use of warfarin in patients with NVAF can be challenging. First, warfarin has narrow therapeutic index, therefore patients may need to attend healthcare facilities regularly for INR monitoring and dose adjustment. Second, the daily maintenance dose of warfarin can vary among patients and in the same patients. Third, the metabolism of warfarin can be affected by certain foods, drugs and alcohol [11]. Fourth, genetic variation has been shown to influence the metabolism of warfarin [12]. In spite of these challenges, warfarin is still a popular oral anticoagulant in NVAF, mainly due to its affordability and availability.

Health-related quality of life (HRQoL) is defined as individuals' satisfaction or happiness with an aspect of life determined by their physical, mental, emotional or social functioning [13]. It is an emerging focus among patients receiving warfarin for NVAF due to the longer life expectancy brought about by the advancement in healthcare. Treatment satisfaction is defined as individuals' rating of important attributes of the process and outcomes of their treatment experience, which involve the interaction of expectation, preference and satisfaction $[14,15]$. The HRQoL of patients on warfarin is frequently impaired due to change in their lifestyle, risk of bleeding, as well as lack of objective symptomatic relief from the medications. At the same time, their HRQoL may also be affected by their treatment satisfaction. Improving and enhancing satisfaction with the treatment regime is an important aspect in management of patients with chronic illness, such as AF [16]. Studies have shown a higher satisfaction, higher adherence, better knowledge and lower apprehension to warfarin therapy is associated with significantly better INR control [17-19].

To date, studies that assess the TTR, HRQoL and treatment satisfaction of patients taking warfarin for NVAF concurrently are still limited. Therefore, this study aims to evaluate the TTR, HRQoL and treatment satisfaction of patients taking warfarin for NVAF in a tertiary cardiology referral center located in Sarawak, a state in Malaysia. The HRQoL and treatment satisfaction of patients were also compared based on their TTR.

\section{Methodology}

\section{Study design}

We conducted a cross-sectional study for patients with NVAF attending the anticoagulant clinic of the Sarawak Heart Center (SHC) from 1st June 2018 to 31st May 2019. All patients included were aged 18 years and above, and have been receiving warfarin for at least past six months. Patients were excluded from the study if they had incomplete INR record, co-existing anti-platelet therapy, crossed-over from direct oral anticoagulant (DOAC), valvular heart disease, mechanical heart valves, hospital admission within one month before the interview attributed to any cause other than complications of warfarin, and unable to answer the questionnaires independently. The estimated minimum sample size for the study was 217 calculated based on the formula of cross sectional study for qualitative variables, $\mathrm{n}=\mathrm{Z}_{1-\alpha}{ }^{2} \times \mathrm{p}(1-\mathrm{p}) / \mathrm{d} 2$, in which reported prevalence of good TTR based on previous study (p) for Asian was $16.7 \%, \mathrm{Z}_{1-\alpha}$ was a constant of 1.96, and precision (d) of 0.05 [20, 21]. We obtained written informed consent from all the participants, and ethics approval from the Medical Research and Ethics Committee of the National Medical Research Registry of Malaysia (NMRR-17-1086-34402) and hospital. This study was conducted in accordance with the Declaration of Helsinki.

\section{Data collection and questionnaires}

We identified the eligible patients from the registry of the hospital and approached them on the day of clinic visit. The demographic and clinical data were obtained via face-to-face interview, hospital case note, home-based card and electronic INR record.

The patients' INR readings over the past six months to a year were recorded. Their TTR was calculated by using Rosendaal technique [22], which exclude the INR readings of the first six weeks after the warfarin was started. The clinical benefit of warfarin was not superior than dual anti-platelet when the TTR was less than 58\% [23]. 
Therefore, in this study, we defined a good TTR at $60 \%$. Hospitalisation refers to any admission due to the complications of taking warfarin, such as bleeding or thromboembolism. The comorbidities only took into account parameters in $\mathrm{CHA}_{2} \mathrm{DS}_{2}$-VASc, namely congestive cardiac failure, hypertension, diabetes mellitus, stroke/ transient ischemic attack/thromboembolism history and vascular disease [9].

Patients were instructed to answer the Short Form 12v2 Health Survey (SF12v2) and Duke Anticoagulant Satisfaction Scale (DASS) independently to determine their HRQoL and treatment satisfaction [24, 25]. These questionnaires were available in original English version, as well as a validated Malay version [26, 27]. Patients could obtain explanation from the investigators if there was any problem with understanding the questionnaires. SF12v2 is a generic questionnaire that evaluates patients' HRQoL in eight domains [24]. These include physical functioning $(\mathrm{PF})$, role physical (RP), bodily pain (BP), general health perceptions $(\mathrm{GH})$, vitality $(\mathrm{V})$, social functioning $(\mathrm{SF})$, role emotional (RE) and mental health $(\mathrm{MH})$. The Quality Metric's Health Outcome ${ }^{\mathrm{TM}}$ Scoring Software 5.0 is used to calculate the physical component summary (PCS) and mental component summary (MCS) from these eight domains. PCS and MCS range from $0-100$, the higher value represent a better HRQoL. PCS tends to decrease with age, while MCS tends to increase with age [24]. DASS is a disease-specific questionnaire containing 25 items that address patients' treatment satisfaction in three sub-dimensions, namely limitations (L), hassles and burdens $(\mathrm{H} \& B)$ and positive psychological impacts (PPI) [25]. The $\mathrm{L}$ and H\&B are also considered as negative impacts of oral anticoagulant. The total score of DASS range from 25 - 175, with lower value represents a better satisfaction. Both SF12v2 and DASS involve comparison between groups, and is not meant to be interpreted alone within a group as a categorical variable.

\section{Analysis}

We presented categorical variables as percentage and continuous variables as the mean \pm standard deviation (SD) or median with interquartile range. For betweengroups differences, Chi-squared test or Fisher's exact test was used for categorical variables, while independent sample t-test or the Mann-Whitney U test was used for continuous variables. ANCOVA was used to analyse HRQoL and treatment satisfaction between groups corrected for significant variables. A $p$ value less than 0.05 was considered statistically significant. The analysis was performed by using Statistical Package for the Social Sciences (SPSS for Windows version 25.0, SPSS Inc., Chicago, IL, USA).

\section{Results}

Patient demographics and clinical characteristics

A total of 300 patients were included in the study (Fig. 1). Their demographic and clinical characteristics are shown in Table 1. Patients were predominantly male (53.4\%), native of Sarawak (53.0\%), married (73.3\%), non-vegetarian/vegan (95.0\%) and non-regular alcohol consumers (98.0\%). Hypertension (58.7\%) was the commonest comorbid, followed by congestive cardiac failure (18.0\%), diabetes mellitus (17.3\%) and stroke (3.7\%). The mean $\mathrm{CHA}_{2} \mathrm{DS}_{2}$-VASc score was $2.0 \pm 1.3$.

\section{TTR}

In this study, patients had been receiving warfarin for a mean duration of $6.3 \pm 6.5$ years. Their mean TTR score was $47.0 \pm 17.3 \%$. Seventy-three $(24.3 \%)$ patients had good TTR, with mean score of $70.2 \pm 8.7 \%$. The mean TTR for remaining 227 (75.5\%) patients were

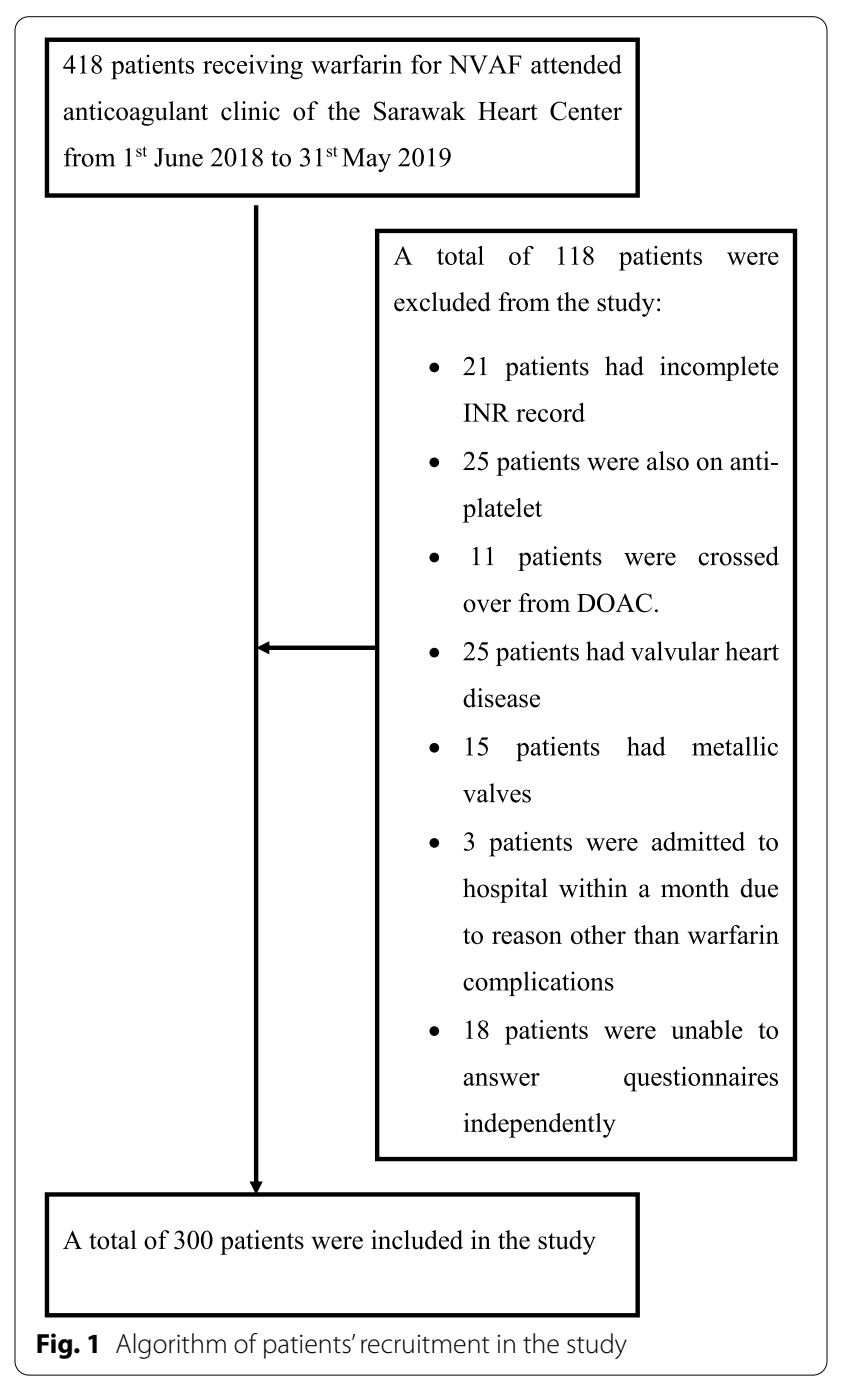


Table 1 Demographic and clinical characteristic of patients on warfarin

\begin{tabular}{|c|c|c|c|c|}
\hline \multirow[t]{2}{*}{ Characteristics } & \multirow[t]{2}{*}{ Total patients, $n=300$} & \multicolumn{2}{|l|}{ Patients TTR (n, \%) } & \multirow[t]{2}{*}{$p$ value } \\
\hline & & $\begin{array}{l}\text { Good } \\
73(24.3)\end{array}$ & $\begin{array}{l}\text { Poor } \\
227(75.7)\end{array}$ & \\
\hline \multicolumn{5}{|l|}{ Age (mean \pm SD; 95\% Cl) } \\
\hline Years & $\begin{array}{l}59.7 \pm 15.6 \\
57.9-61.5\end{array}$ & $57.2 \pm 15.0 ; 53.7-60.7$ & $\begin{array}{l}60.5 \pm 15.7 \\
58.5-62.6\end{array}$ & 0.559 \\
\hline \multicolumn{5}{|l|}{ Gender $(n, \%)$} \\
\hline Male & $172(57.3)$ & 39 (53.4) & $133(58.6)$ & 0.438 \\
\hline Female & $128(42.7)$ & $34(46.6)$ & $94(41.4)$ & \\
\hline \multicolumn{5}{|l|}{ Ethnicity $(\mathrm{n}, \%)$} \\
\hline Malay & $67(22.3)$ & $15(20.5)$ & $52(22.9)$ & 0.095 \\
\hline Chinese & $68(22.7)$ & $32(43.8)$ & $44(19.4)$ & \\
\hline Native & $159(53.0)$ & $2(2.7)$ & $127(55.9)$ & \\
\hline Others & $6(2.0)$ & $2(1.8)$ & $4(1.8)$ & \\
\hline \multicolumn{5}{|l|}{ Partner status (n, \%) } \\
\hline No partner & $80(26.7)$ & $18(24.7)$ & $62(27.3)$ & 0.420 \\
\hline With partner & $220(73.3)$ & $55(75.3)$ & $165(72.7)$ & \\
\hline \multicolumn{5}{|l|}{ Education (n, \%) } \\
\hline None & $58(19.3)$ & $6(8.2)$ & $52(22.9)$ & 0.001 \\
\hline Primary & $101(33.7)$ & $19(26.0)$ & $82(36.1)$ & \\
\hline Secondary & $115(38.3)$ & $41(56.2)$ & $74(32.6)$ & \\
\hline College/Tertiary & $26(8.7)$ & $7(9.6)$ & $19(8.4)$ & \\
\hline \multicolumn{5}{|l|}{ Occupation (n, \%) } \\
\hline Unemployed & $147(49.0)$ & $30(41.1)$ & $117(51.5)$ & 0.269 \\
\hline Government dependent/ pensioner & $72(24.0)$ & $19(26.0)$ & $53(23.3)$ & \\
\hline Private & $81(27.0)$ & $24(32.9)$ & $57(25.1)$ & \\
\hline \multicolumn{5}{|l|}{ Diet $(n, \%)$} \\
\hline Non-vegetarian/vegan & $285(95.0)$ & $69(94.5)$ & $216(95.2)$ & 0.829 \\
\hline Vegetarian/vegan & $15(5.0)$ & $4(5.5)$ & $11(4.8)$ & \\
\hline \multicolumn{5}{|l|}{ Alcohol $(n, \%)$} \\
\hline No & $294(98.0)$ & $71(97.3)$ & $223(98.2)$ & 0.604 \\
\hline Yes & $6(2.0)$ & $2(2.7)$ & $4(1.8)$ & \\
\hline \multicolumn{5}{|l|}{ Comorbidities ( $n, \%)$} \\
\hline Congestive cardiac failure* & $54(18.0)$ & $16(21.9)$ & $38(16.7)$ & 0.317 \\
\hline Hypertension* & $176(58.7)$ & $36(49.3)$ & $140(61.7)$ & 0.062 \\
\hline Diabetes mellitus* & $52(17.3)$ & $11(15.1)$ & $41(18.1)$ & 0.557 \\
\hline Stroke/TIA//thromboembolism* & $11(3.7)$ & $6(8.2)$ & $5(2.2)$ & 0.017 \\
\hline Vascular disease $e^{*}$ & 0 & 0 & 0 & NA \\
\hline Others* & $67(22.3)$ & $12(16.4)$ & $55(24.2)$ & 0.164 \\
\hline \multicolumn{5}{|l|}{$\mathrm{CHA}_{2} \mathrm{DS}_{2}$-VASC } \\
\hline None & $\begin{array}{l}2.0 \pm 1.3 \\
1.8-2.1\end{array}$ & $\begin{array}{l}1.8 \pm 1.1 \\
1.6-2.1\end{array}$ & $\begin{array}{l}2.0 \pm 1.4 \\
1.9-2.2\end{array}$ & 0.204 \\
\hline \multicolumn{5}{|l|}{ Treatment duration (mean \pm SD; 95\% Cl) } \\
\hline Years & $\begin{array}{l}6.3 \pm 6.5 \\
5.5-7.0\end{array}$ & $\begin{array}{l}7.8 \pm 8.3 \\
5.9-9.8\end{array}$ & $\begin{array}{l}5.7 \pm 5.7 \\
5.0-6.5\end{array}$ & $<0.001$ \\
\hline \multicolumn{5}{|l|}{ TTR (mean \pm SD; 95\% Cl) } \\
\hline$\%$ & $\begin{array}{l}47.0 \pm 17.3 \\
45.0-48.9\end{array}$ & $\begin{array}{l}70.2 \pm 8.7 \\
68.2-72.2\end{array}$ & $\begin{array}{l}39.5 \pm 11.9 \\
38.0-41.1\end{array}$ & 0.006 \\
\hline \multicolumn{5}{|l|}{ Hospitalization (n, \%) } \\
\hline No & $245(81.7)$ & $58(79.5)$ & $187(82.4)$ & 0.574 \\
\hline Yes & $55(18.3)$ & $15(20.5)$ & $40(17.6)$ & \\
\hline
\end{tabular}


Table 1 (continued)

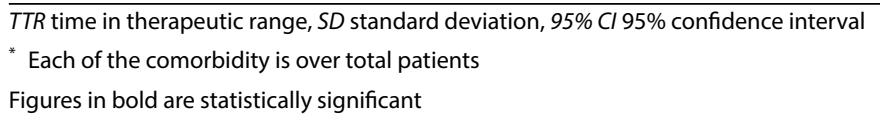

significantly lower, $39.5 \pm 11.9 \%(p=0.006)$. Patients with good TTR had been receiving warfarin therapy for a significantly longer duration (mean, $7.8 \pm 8.3$ years versus $5.7 \pm 5.7$ years, $p<0.001)$. The education level was significantly different between patients with good and poor TTR $(p=0.001) .22 .9 \%$ of patients with poor TTR were uneducated, while only $8.2 \%$ of patients with good TTR did not attend school. Even though stroke/TIA/thromboembolism was significantly more common in patients with good TTR ( $8.2 \%$ vs. $2.2 \%, p=0.017)$, the number of these patients was too small. Otherwise, there was no different in terms of patients' gender, ethnicity, marital status, occupation, diet preference, alcohol intake and other comorbidities between patient with good and poor TTR.

QOL

The overall PCS and MCS score were 47.0 \pm 9.0 and $53.5 \pm 9.6$, respectively (Table 2 ). There was no significant difference in the score of PCS $(48.3 \pm 8.7$ vs. $46.5 \pm 9.1, p=0.150)$, MCS $(53.4 \pm 8.6$ vs.53.6 \pm 9.7 , $p=0.919)$ and each domain of SF-12v2 $(p=0.184-0.684)$ between patients with good and poor TTR, except for SF $(51.5 \pm 7.9$ vs. $48.2 \pm 11.3, p=0.019)$. The scores for PCS $(48.2 \pm 1.1$ vs. $46.6 \pm 0.6, p=0.175 ; 47.7 \pm 1.1$ versus $46.7 \pm 0.6, p=0.395)$, MCS ( $53.5 \pm 1.1$ vs. $53.5 \pm 0.6$, $p=0.998 ; 53.6 \pm 1.1$ vs. $53.5 \pm 0.6, p=0.951)$ and each domain of SF-12v2 ( $p=0.207-0.737 ; p=0.069-0.968)$ remained not significantly different between patients with good and poor TTR even after adjusted for treatment duration and education level, except for SF adjusted for treatment duration $(51.9 \pm 1.3$ versus $48.1 \pm 0.7$, $p=0.014)$.

\section{Treatment satisfaction:}

The total score for DASS was $55.2 \pm 21.3$, while the score for $\mathrm{L}, \mathrm{H} \& \mathrm{~B}$, as well as PPI were $18.0 \pm 10.0,15.6 \pm 9.1$

Table 2 Comparison of SF-12v2 score between patients with good and poor TTR

\begin{tabular}{|c|c|c|c|c|c|c|c|c|c|c|}
\hline \multirow{3}{*}{$\begin{array}{l}\text { SF12v2 } \\
\text { parameters }\end{array}$} & \multirow{3}{*}{$\begin{array}{l}\text { Total score; } \\
\text { Mean } \pm \text { SD; } \\
95 \% \mathrm{Cl}\end{array}$} & \multicolumn{3}{|c|}{ Without adjustment } & \multicolumn{3}{|c|}{ Adjusted for duration* } & \multicolumn{3}{|c|}{ Adjusted for education* } \\
\hline & & & & $p$ value & & & $p$ value & & & $p$ value \\
\hline & & $\begin{array}{l}\text { Mean } \pm \text { SD; } \\
95 \% \mathrm{Cl}\end{array}$ & $\begin{array}{l}\text { Mean } \pm \text { SD; } \\
95 \% \mathrm{Cl}\end{array}$ & & $\begin{array}{l}\text { Mean } \pm \text { SE; } \\
95 \% \mathrm{Cl}\end{array}$ & $\begin{array}{l}\text { Mean } \pm \text { SE; } \\
95 \% \mathrm{Cl}\end{array}$ & & $\begin{array}{l}\text { Mean } \pm S E ; \\
95 \% \mathrm{Cl}\end{array}$ & $\begin{array}{l}\text { Mean } \pm \text { SE; } \\
95 \% \mathrm{Cl}\end{array}$ & \\
\hline PCS & $\begin{array}{l}47.0 \pm 9.0 \\
45.9-48.0\end{array}$ & $\begin{array}{l}48.3 \pm 8.7 \\
46.3-50.3\end{array}$ & $\begin{array}{l}46.5 \pm 9.1 \\
45.3-47.7\end{array}$ & 0.150 & $\begin{array}{l}48.2 \pm 1.1 \\
46.1-50.3\end{array}$ & $\begin{array}{l}46.6 \pm 0.6 \\
45.4-47.7\end{array}$ & 0.175 & $\begin{array}{l}47.7 \pm 1.1 \\
45.7-49.8\end{array}$ & $\begin{array}{l}46.7 \pm 0.6 \\
45.5-47.9\end{array}$ & 0.395 \\
\hline MCS & $\begin{array}{l}53.5 \pm 9.6 \\
52.4-54.6\end{array}$ & $\begin{array}{l}53.4 \pm 8.6 \\
51.4-55.4\end{array}$ & $\begin{array}{l}53.6 \pm 9.7 \\
52.3-54.9\end{array}$ & 0.919 & $\begin{array}{l}53.5 \pm 1.1 \\
51.3-55.8\end{array}$ & $\begin{array}{l}53.5 \pm 0.6 \\
52.3-54.8\end{array}$ & 0.998 & $\begin{array}{l}53.6 \pm 1.1 \\
51.3-55.9\end{array}$ & $\begin{array}{l}53.5 \pm 0.6 \\
52.2-54.8\end{array}$ & 0.951 \\
\hline PF & $\begin{array}{l}47.0 \pm 10.6 \\
45.8-48.2\end{array}$ & $\begin{array}{l}48.0 \pm 10.9 \\
45.5-50.6\end{array}$ & $\begin{array}{l}46.7 \pm 10.5 \\
45.3-48.1\end{array}$ & 0.358 & $\begin{array}{l}47.9 \pm 1.2 \\
45.5-50.4\end{array}$ & $\begin{array}{l}46.7 \pm 0.7 \\
45.3-48.1\end{array}$ & 0.399 & $\begin{array}{l}47.4 \pm 1.2 \\
45.0-49.9\end{array}$ & $\begin{array}{l}46.9 \pm 0.7 \\
45.5-48.3\end{array}$ & 0.704 \\
\hline $\mathrm{RP}$ & $\begin{array}{l}48.2 \pm 9.6 \\
47.1-49.3\end{array}$ & $\begin{array}{l}48.6 \pm 8.8 \\
46.5-50.6\end{array}$ & $\begin{array}{l}48.1 \pm 9.9 \\
46.8-49.4\end{array}$ & 0.684 & $\begin{array}{l}48.6 \pm 1.1 \\
46.3-50.8\end{array}$ & $\begin{array}{l}48.1 \pm 0.6 \\
46.8-49.3\end{array}$ & 0.698 & $\begin{array}{l}48.2 \pm 1.1 \\
46.0-50.5\end{array}$ & $\begin{array}{l}48.2 \pm 0.6 \\
46.9-49.4\end{array}$ & 0.968 \\
\hline $\mathrm{BP}$ & $\begin{array}{l}49.6 \pm 10.5 \\
48.5-50.8\end{array}$ & $\begin{array}{l}51.1 \pm 9.5 \\
48.8-53.3\end{array}$ & $\begin{array}{l}49.2 \pm 10.7 \\
47.8-50.6\end{array}$ & 0.184 & $\begin{array}{l}51.0 \pm 1.2 \\
48.6-53.4\end{array}$ & $\begin{array}{l}49.2 \pm 0.7 \\
47.8-50.6\end{array}$ & 0.207 & $\begin{array}{l}50.5 \pm 1.2 \\
48.0-52.9\end{array}$ & $\begin{array}{l}49.4 \pm 0.7 \\
48.0-50.7\end{array}$ & 0.446 \\
\hline $\mathrm{GH}$ & $\begin{array}{l}47.6 \pm 9.7 \\
46.5-48.7\end{array}$ & $\begin{array}{l}47.9 \pm 9.5 \\
45.7-50.1\end{array}$ & $\begin{array}{l}47.5 \pm 9.8 \\
46.2-48.8\end{array}$ & 0.730 & $\begin{array}{l}47.9 \pm 1.2 \\
45.7-50.2\end{array}$ & $\begin{array}{l}47.5 \pm 0.7 \\
46.2-48.8\end{array}$ & 0.737 & $\begin{array}{l}48.1 \pm 1.2 \\
45.8-50.4\end{array}$ & $\begin{array}{l}47.4 \pm 0.7 \\
46.2-48.7\end{array}$ & 0.639 \\
\hline V & $\begin{array}{l}56.3 \pm 11.3 \\
55.0-57.6\end{array}$ & $\begin{array}{l}56.9 \pm 11.5 \\
54.2-59.6\end{array}$ & $\begin{array}{l}56.1 \pm 11.3 \\
54.6-57.6\end{array}$ & 0.602 & $\begin{array}{l}57.0 \pm 1.3 \\
54.3-59.6\end{array}$ & $\begin{array}{l}56.1 \pm 0.8 \\
54.6-57.5\end{array}$ & 0.561 & $\begin{array}{l}57.1 \pm 1.3 \\
54.5-59.7\end{array}$ & $\begin{array}{l}56.0 \pm 0.8 \\
54.5-57.5\end{array}$ & 0.505 \\
\hline SF & $\begin{array}{l}49.0 \pm 10.7 \\
47.8-50.2\end{array}$ & $\begin{array}{l}51.5 \pm 7.9 \\
49.7-53.4\end{array}$ & $\begin{array}{l}48.2 \pm 11.3 \\
46.7-49.6\end{array}$ & 0.019 & $\begin{array}{l}51.9 \pm 1.3 \\
49.2-54.2\end{array}$ & $\begin{array}{l}48.1 \pm 0.7 \\
46.7-49.5\end{array}$ & 0.014 & $\begin{array}{l}51.0 \pm 1.2 \\
48.5-53.4\end{array}$ & $\begin{array}{l}48.3 \pm 0.7 \\
47.0-49.7\end{array}$ & 0.069 \\
\hline $\mathrm{RE}$ & $\begin{array}{l}48.8 \pm 10.7 \\
47.6-50.0\end{array}$ & $\begin{array}{l}48.2 \pm 10.7 \\
45.7-50.7\end{array}$ & $\begin{array}{l}49.0 \pm 10.7 \\
47.6-50.4\end{array}$ & 0.563 & $\begin{array}{l}48.1 \pm 1.3 \\
45.6-50.6\end{array}$ & $\begin{array}{l}49.0 \pm 0.7 \\
47.6-50.4\end{array}$ & 0.523 & $\begin{array}{l}48.2 \pm 1.2 \\
45.7-50.7\end{array}$ & $\begin{array}{l}49.0 \pm 0.7 \\
47.6-50.4\end{array}$ & 0.607 \\
\hline $\mathrm{MH}$ & $\begin{array}{l}53.8 \pm 9.9 \\
52.7-55.0\end{array}$ & $\begin{array}{l}53.4 \pm 9.3 \\
51.2-55.5\end{array}$ & $\begin{array}{l}54.0 \pm 10.1 \\
52.7-55.3\end{array}$ & 0.634 & $\begin{array}{l}53.5 \pm 1.2 \\
51.2-55.8\end{array}$ & $\begin{array}{l}54.0 \pm 0.7 \\
52.7-55.3\end{array}$ & 0.714 & $\begin{array}{l}53.4 \pm 1.2 \\
51.1-55.7\end{array}$ & $\begin{array}{l}54.0 \pm 0.7 \\
52.7-55.3\end{array}$ & 0.661 \\
\hline
\end{tabular}

SF12v2 short form 12v2 health survey, SD standard deviation, 95\% Cl 95\% confidence interval, PCS physical component summary, MCS mental component summary, $P F$ physical functioning, $R P$ role-physical, $B P$ bodily pain, $G H$ general health perceptions, $V$ vitality, $S F$ social functioning, $R E$ role-emotional, $M H$ mental health * Adjusted with ANCOVA test

Figures in bold are statistically significant 
Table 3 Comparison of DASS score between patients with good and poor TTR

\begin{tabular}{|c|c|c|c|c|c|c|c|c|c|}
\hline \multirow{3}{*}{$\begin{array}{l}\text { DASS } \\
\text { parameters }\end{array}$} & \multirow{3}{*}{$\begin{array}{l}\text { Total score, } \\
\text { Mean } \pm \text { SD; } \\
95 \% \mathrm{Cl}\end{array}$} & \multicolumn{3}{|c|}{ Without adjustment } & \multicolumn{2}{|c|}{ Adjusted for duration* } & \multicolumn{3}{|c|}{ Adjusted for education* } \\
\hline & & Good TTF & Poor TTF & $p$ value & Good TTF & Poor TTF & Good TTF & Poor TTF & $p$ value \\
\hline & & $\begin{array}{l}\text { Mean } \pm \text { SD; } \\
95 \% \mathrm{Cl}\end{array}$ & $\begin{array}{l}\text { Mean } \pm \text { SD; } \\
95 \% \mathrm{Cl}\end{array}$ & & $\begin{array}{l}\text { Mean } \pm \text { SE; } \\
95 \% \mathrm{Cl}\end{array}$ & $\begin{array}{l}\text { Mean } \pm \text { SE; } \\
95 \% \mathrm{Cl}\end{array}$ & $\begin{array}{l}\text { Mean } \pm \text { SE; } \\
95 \% \mathrm{Cl}\end{array}$ & $\begin{array}{l}\text { Mean } \pm \text { SE; } \\
95 \% \mathrm{Cl}\end{array}$ & \\
\hline Total & $\begin{array}{l}55.2 \pm 21.3 \\
52.6-57.6\end{array}$ & $\begin{array}{l}54.6 \pm 21.9 \\
49.5-59.7\end{array}$ & $\begin{array}{l}55.4 \pm 21.2 \\
52.6-58.1\end{array}$ & 0.779 & $\begin{array}{l}55.0 \pm 2.5 \\
50.0-59.9\end{array}$ & $\begin{array}{l}55.2 \pm 1.4 ; 0.922 \\
52.5-58.0\end{array}$ & $\begin{array}{l}53.9 \pm 2.5 \\
48.9-58.9\end{array}$ & $\begin{array}{l}55.6 \pm 1.4 \\
52.8-58.4\end{array}$ & 0.563 \\
\hline L & $\begin{array}{l}18.0 \pm 10.0 \\
16.9-19.1\end{array}$ & $\begin{array}{l}18.4 \pm 10.5 \\
15.9-20.8\end{array}$ & $\begin{array}{l}17.9 \pm 9.8 \\
16.6-19.1\end{array}$ & 0.699 & $\begin{array}{l}18.5 \pm 1.2 \\
16.2-20.8\end{array}$ & $\begin{array}{l}17.8 \pm 0.7 ; 0.998 \\
16.5-19.1\end{array}$ & $\begin{array}{l}17.8 \pm 1.2 \\
15.5-20.1\end{array}$ & $\begin{array}{l}18.0 \pm 0.7 \\
16.7-19.3\end{array}$ & 0.864 \\
\hline$H \& B$ & $\begin{array}{l}15.6 \pm 9.1 \\
14.5-16.6\end{array}$ & $\begin{array}{l}14.9 \pm 8.4 \\
13.0-16.9\end{array}$ & $\begin{array}{l}15.8 \pm 9.4 \\
14.5-17.0\end{array}$ & 0.502 & $\begin{array}{l}15.1 \pm 1.1 \\
13.0-17.3\end{array}$ & $\begin{array}{l}15.7 \pm 0.6 ; 0.655 \\
14.5-16.9\end{array}$ & $\begin{array}{l}14.8 \pm 1.1 \\
12.6-16.9\end{array}$ & $\begin{array}{l}15.8 \pm 0.6 \\
14.6-17.0\end{array}$ & 0.419 \\
\hline PPI & $\begin{array}{l}21.6 \pm 5.9 \\
21.0-22.3\end{array}$ & $\begin{array}{l}21.3 \pm 6.3 \\
19.8-22.7\end{array}$ & $\begin{array}{l}21.8 \pm 5.8 \\
21.0-22.5\end{array}$ & 0.531 & $\begin{array}{l}21.3 \pm 0.7 \\
19.9-22.7\end{array}$ & $\begin{array}{l}21.7 \pm 0.4 ; 0.612 \\
21.0-22.5\end{array}$ & $\begin{array}{l}21.3 \pm 0.7 \\
19.9-22.7\end{array}$ & $\begin{array}{l}21.7 \pm 0.4 \\
21.0-22.5\end{array}$ & 0.589 \\
\hline
\end{tabular}

DASS Duke Anticoagulant Satisfaction Scale, SD standard deviation, $95 \% \mathrm{Cl} 95 \%$ confidence interval, $L$ limitations, $H \& B$ hassles and burdens, $P P I$ positive psychological impacts

* Adjusted with ANCOVA test

and 21.6 \pm 5.9 , respectively (Table 3 ). The total DASS score was not significantly different between patients with good or poor TTR $(54.6 \pm 21.9$ vs. $55.4 \pm 21.2$, $p=0.779)$, even after adjusted for treatment duration ( $55.0 \pm 2.5$ vs. $55.2 \pm 1.4, p=0.922)$ and education level $(53.9 \pm 2.5$ vs. $55.6 \pm 1.4, p=0.563)$. Similar non-significant difference was also reported in all the three DASS sub dimensions ( $p=0.502-0.699)$, even after adjusted for treatment duration $(p=0.612-0.998)$ and education level $(p=0.419-0.864)$.

\section{Hospitalisation and complications:}

Fifty-five (18.3\%) patients had been admitted to hospital due to complications of warfarin, all attributed to bleeding tendency. The hospitalisation rate was not significantly different between patients with good and poor $\operatorname{TTR}(20.5 \%$ vs. $17.6 \%, p=0.574)$.

\section{Discussion}

In the present study, only a quarter of patients had good TTR. Higher education level is the independent predictor of better TTR. The HRQoL of these patients was moderate, but their treatment satisfaction was good. The HRQoL and treatment satisfaction was similar irrespective of patients' TTR. The only exception was patients with good TTR tends to have a better social functioning. Nearly one-fifth of patients had been hospitalised for bleeding tendency. Hospitalisations and bleeding tendency were independent of patients' TTR.

Existing studies concluded patients from western countries had better TTR than their Asian counterpart. The International Study of Anticoagulant Management (INSAM) was a retrospective analysis of the real-world patients receiving warfarin for NVAF in five western countries, namely the United State, Canada, France, Italy and Spain [28]. The reported mean TTR was 57.0-68.9\%, while the proportion of patients with good TTR $(\geq 60 \%)$ was $47.7-75.1 \%$. The Global Anticoagulant Registry in the Field (GARFIELD) AF reported an overall mean TTR of $55.4 \%$ and overall good TTR ( $\geq 65 \%$ ) of $41.1 \%$ among their patients [21]. The subgroup analysis however demonstrated only $16.7 \%$ of Asian cohort had good TTR compare to $49.4 \%$ of European cohort. In another study by Chan et al., Hong Kong patients on warfarin for NVAF were reported to have a mean TTR of $38.8 \%$ and good TTR ( $\geq 65 \%$ ) of $14.8 \%$ [29]. Oh et al. reported only $27 \%$ of Korean on warfarin for NVAF had good TTR $(\geq 60 \%)$ [30]. Therefore, the lower mean TTR and the fewer good TTR among patients in this study were similar to other Asia studies. The possible explanations for this variation include Asian may have different diet and genetic, such as polymorphisms of VKOR1 and CYP2C9 genes [11, 12, 31]. The INSAM study and a meta-analysis by van Walraven et al. reported the clinical setting of warfarin given was the strongest predictor of patients TTR, being highest in randomised control trials, followed by anticoagulant clinic and community practise [28, 32]. The present study further added low education level was associated with a poorer TTR.

Regardless of the TTR, patients receiving warfarin for NVAF in the present study have markedly lower score in each domain of SF-12v2 (good TTR: 47.9-56.9; poor TTR: 46.5-56.1) when compare to Malaysia general population (SF-36, 66.7-86.0) [33], therefore significates a poorer HRQoL. To date, there is no published data that compares the HRQoL of adult patients on warfarin for NVAF based on their TTR. Most of the existing studies either compare the HRQoL of these patients versus those on DOAC, or in a longitudinal manner. $\mathrm{Ng}$ et al. [34], Benzimra et al. [35], Contreras et al. [36] and 
Alegret et al. [37] have reported no significant difference in the HRQoL of patients with NVAF receiving longterm warfarin versus DOAC in real-world scenario. On the other hand, Balci et al. reported significant improvement in every domain of SF-12v2 (all $p<0.001$ ) [38], while de Caterina et al. reported significant improvement in severe mobility problem $(p=0.003)$, pain/discomfort $(p=0.035)$, and anxiety/depression $(p<0.001)$ of EuroQoL Instrument 5 levels (EQ-5D-5L) among NVAF patients after switching from warfarin to DOAC [39]. The patients in the current study had slightly higher value in each domain of the SF-12v2 except SF, but much lower mean TTR (47.0\% vs. $54.9 \%)$ and proportion with good TTR $(24.3 \%$ vs. $45.0 \%)$ when compared to similar subgroup of patients reported in Ng et al. [34]. Both studies were conducted in the anticoagulant clinic of a tertiary hospital, but the latter located in the Peninsular of Malaysia. The better social functioning among patients with good TTR in the current study could be due to their higher education level.

In this study, the finding of treatment satisfaction was independent of patients' TTR was consistent with that reported in previous study. In the Outcome Registry for Better Informed Treatment of AF (ORBIT-AF) study, the treatment satisfaction of patients on warfarin for AF evaluated by using Anti-Clot Treatment scale (ACTS) was not affected by the TTR [40]. Similar finding was also observed in the Korean patients who received warfarin for NVAF when assessed by the Treatment Satisfaction Questionnaire for Medication (TSQM) [30]. A post-hoc study (ALADIN and ESPARTA) however reported significantly higher ACTS burden scale (better satisfaction) in NVAF patients with good TTR $(\geq 50 \%)(p=0.024)$ [19]. The cut-off point of good TTR in this study was lower than that commonly used (60-65\%), therefore unable to make direct comparison with the current study.

The current study concludes that majority of the NVAF patients attending SHC had poor anticoagulant control, which may be attributed to lower education level. We recommend patients with poor TTR should be reassured that achieving a good TTR confers a better clinical outcome but does not compromise their HRQoL or satisfaction. These patients may benefit from frequent education on anticoagulant by clinicians and pharmacists during their follow-up appointments. Furthermore, the implementation of warfarin medication therapy adherence clinic (WMTAC) protocol may also help to improve patients' INR control [41]. Switching from warfarin to DOAC should be consider in NVAF patients with poor TTR in view of the better efficacy, HRQoL and treatment satisfaction, as well as lesser side-effects. In area where the poor INR control among NVAF patients is common, DOAC should be consider as the first-line anticoagulant.
To our knowledge, this is the first study that compares the HRQoL of NVAF patients on long-term warfarin based on their TTR. Analysis of each domain of the SF12v2 and each item of the DASS were performed in order to provide a more detailed comparison. The analyses of HRQoL and treatment satisfaction were adjusted for treatment duration and education level as cofounders to minimise the result bias. This study had several limitations. Firstly, it was performed in a single centre, thus limiting the generalisability of the results. Secondly, the cross-sectional design might not be able to completely reflect the HRQoL, as HRQoL may vary over time. Thirdly, the patients HAS-BLED score and severity of bleeding was not assessed. Most of the patients were unable to remember their non-clinical significant minor bleed episode. Fourthly, patients with vascular disease taking anti-platelet were excluded. Fifthly, the TTR was calculated by using Rosendaal technique only, without comparison to the traditional method. The former assumes the changes between consecutive INR measurements are linear over time, while the latter only consider the INR value at a point of time. Finally, the treatment complications were subject to the recall bias of patients, but this was minimised by double checking available medical records.

\section{Conclusion}

Majority of the patients on long-term warfarin for NVAF in the current study have poor TTR. Their HRQoL and treatment satisfaction are independent of their TTR. Achieving a good TTR do not compromise the HRQOL and treatment satisfaction. Therefore, appropriate measures should be taken to optimise INR control, failing which DOAC therapy should be considered. Self-management of warfarin can improve patients' HRQoL, while adequate anticoagulant education that promote better understanding of different dosage forms, drug regime and potential side-effects can improve their treatment satisfaction.

\section{Abbreviations}

AF: Atrial fibrillation; NVAF: Non-valvular AF; INR: International normalised ratio; TTR: Time in therapeutic range; HRQoL: Health-related quality of life; SHC: Sarawak Heart Center; DOAC: Direct oral anticoagulants; TIA: Transient ischemic attack; SF12v2: Short Form 12v2 Health Survey; DASS: Duke Anticoagulant Satisfaction Scale; PF: Physical functioning; RP: Role physical; BP: Bodily pain; GH: General health perceptions; V: Vitality; SF: Social functioning; RE: Role emotional; MH: Mental health; PCS: Physical component summary; MCS: Mental component summary; L: Limitations; H\&B: Hassles and burdens; PPI: Positive psychological impacts; SD: Standard deviation; INSAM: The International Study of Anticoagulant Management; GARFILED: The Global Anticoagulant Registry in the Field; EQ-5D-5L: EuroQoL Instrument 5 levels; ORBIT-AF: Outcome Registry for Better Informed Treatment of AF; ACTS: AntiClot Treatment scale (ACTS); TSQM: Treatment Satisfaction Questionnaire for Medication; WMTAC: Warfarin medication therapy adherence clinic. 


\section{Acknowledgements}

We would like to thank the Director General of Health Malaysia for his permission to publish this article. We want to express our gratitude to all the patients who had participated in the study.

\section{Authors' contributions}

Diana-Leh-Ching Ng, Natasya Marliana Bt Abdul Malik, Chee-Shee Chai, Greta-Miranda-Kim-Choo Goh, Seng-Beng Tan, Ping-Chong Bee, Gin-Gin Gan, and Asri B Said had contributed substantially to this study, which includes: 1. Substantial contributions to conception and design, data acquisition, or data analysis and interpretation; 2. Drafting the article or critically revising it for important intellectual content; 3 . Final approval of the version to be published; and 4. Agreement to be accountable for all aspects of the work in ensuring that questions related to the accuracy or integrity of the work are appropriately investigated and resolved. All authors read and approved the final manuscript.

\section{Funding}

This study was fully supported by the UNIMAS Special Grant Scheme with the Grant Number F05/SpGS/1550/2017.

\section{Availability of data and materials}

The datasets used and/or analysed during the current study are available from the corresponding author on reasonable request.

\section{Ethic approval and informed consent}

Ethical approval for this study was obtained from the Medical Research and Ethics Committee (MREC), Ministry of Health Malaysia. Written informed consent was obtained from all the study patients.

\section{Consent for publication}

Not applicable.

\section{Competing interests}

The authors declare no potential conflicts of interest in respect to the research, authorship, and publication of this article.

\section{Author details}

1 Department of Medicine, Faculty of Medicine and Health Science, University Malaysia Sarawak, Kota Samarahan, Sarawak, Malaysia. ${ }^{2}$ Department of Nursing, Faculty of Medicine and Health Science, University Malaysia Sarawak, Kota Samarahan, Sarawak, Malaysia. ${ }^{3}$ Department of Medicine, Faculty of Medicine, University of Malaya, Kuala Lumpur, Malaysia.

Received: 13 July 2020 Accepted: 13 October 2020

Published online: 20 October 2020

\section{References}

1. Wyndham CR. Atrial fibrillation: the most common arrhythmia. Tex Heart Inst J. 2000;27(3):257-67.

2. Chugh SS, Havmoeller R, Narayanan K, Singh D, Rienstra M, Benjamin E $J_{,}$ et al. Worldwide epidemiology of atrial fibrillation: a Global Burden of Disease 2010 Study. Circulation. 2014;129(8):837-47.

3. Magnani JW, Wang N, Benjamin EJ, Garcia ME, Bauer DC, Butler J, et al. Atrial fibrillation and declining physical performance in older adults: the health, aging, and body composition study. Circ Arrhythm Electrophysiol. 2016;9(5):e003525.

4. Wolf PA, Abbott RD, Kannel WB. Atrial fibrillation as an independent risk factor for stroke: the Framingham Study. Stroke. 1991;22(8):983-8.

5. Lin HJ, Wolf PA, Benjamin EJ, Belanger AJ, D'Agostino RB. Newly diagnosed atrial fibrillation and acute stroke. Framingham Study Stroke. 1995;26(9):1527-30

6. Pearce $L A$, Hart RG, Halperin JL. Assessment of three schemes for stratifying stroke risk in patients with nonvalvular atrial fibrillation. Am J Med. 2000;109(1):45-51.

7. Rahman F, Kwan GF, Benjamin EJ. Global epidemiology of atrial fibrillation. Nat Rev Cardiol. 2014;1 1(11):639-54.
8. Fauchier L, Philippart R, Clementy N, Bourguignon T, Angoulvant D, Ivanes $F$, et al. How to define valvular atrial fibrillation? Arch Cardiovasc Dis. 2015;108(10):530-9.

9. Lip GYH, Banerjee A, Boriani G, Chiang C, Fargo R, Freedman B, et al. Antithrombotic therapy for atrial fibrillation: chest guideline and expert panel report. Chest. 2018;154(5):1121-201.

10. White HD, Gruber M, Feyzi J, Kaatz S, Tse HF, Husted S, et al. Comparison of outcomes among patients randomized to warfarin therapy according to anticoagulant control: results from SPORTIF III and V. Arch Intern Med. 2007;167(3):239-45

11. Holbrook AM, Pereira JA, Labiris R, et al. Systematic overview of warfarin and its drug and food interactions. Arch Intern Med. 2005;165(10):1095-106.

12. Wadelius M, Chen LY, Eriksson N, Bumpstead S, Ghori J, Wadelius C, et al. Association of warfarin dose with genes involved in its action and metabolism. Hum Genet. 2007;121(1):23-34.

13. Ware JE Jr, Sherbourne CD. The MOS 36-item short-form health survey (SF-36). I. Conceptual framework and item selection. Med Care. 1992;30(6):473-83.

14. Weaver M, Patrick DL, Markson LE, Martin D, Frederic I, Berger M. Issues in the measurement of satisfaction with treatment. Am J Manag Care. 1997;3(4):579-94.

15. Kravitz RL. Patients' expectations for medical care: an expanded formulation based on review of the literature. Med Care Res Rev: MCRR. 1996;53(1):3-27.

16. Bartlett EE, Grayson M, Barker R, Levine DM, Golden A, Libber S. The effects of physician communications skills on patient satisfaction; recall, and adherence. J Chronic Dis. 1984;37(9):755-64.

17. Wang Y, Kong MC, Lee LH, Ng HJ, Ko Y. Knowledge, satisfaction, and concerns regarding warfarin therapy and their association with warfarin adherence and anticoagulation control. Thromb Res. 2014;133(4):550-4.

18. Balkhi B, Al-Rasheedi M, Elbur Al, Alghamadi A. Association between satisfaction with and adherence to warfarin therapy on the control of international normalized ratio: A hospital-based study in Saudi Arabia. Saudi Pharm J. 2018;26(1):145-9.

19. Suárez Fernández C, Castilla-Guerra L, Cantero Hinojosa J, Suriñach JM, Acosta de Bilbao F, Tamarit JJ, et al. Satisfaction with oral anticoagulants in patients with atrial fibrillation. Patient preference and adherence. 2018;12:267-74.

20. Charan J, Biswas T. How to calculate sample size for different study designs in medical research? Indian J Psychol Med. 2013;35(2):121-6.

21. Haas S, Ten Cate H, Accetta G, Angchaisuksiri P, Bassand J-P, Camm AJ, et al. Quality of Vitamin K antagonist control and 1-year outcomes in patients with atrial fibrillation: a global perspective from the GARFIELDAF registry. PloS One. 2016;11(10):e0164076.

22. Rosendaal FR, Cannegieter SC, van der Meer FJ, Briët E. A method to determine the optimal intensity of oral anticoagulant therapy. Thromb Haemost. 1993;69(3):236-9.

23. Connolly SJ, Pogue J, Eikelboom J, Flaker G, Commerford P, Franzosi MG et al. Benefit of oral anticoagulant over antiplatelet therapy in atrial fibrillation depends on the quality of international normalized ratio control achieved by centers and countries as measured by time in therapeutic range. Circulation. 2008;118(20):2029-37.

24. Ware JE, Kosinski M, Keller SD. A 12-item short-form health survey: construction of scales and preliminary tests of reliability and validity. Med Care. 1996;34(3):220-33.

25. Samsa G, Matchar DB, Dolor RJ, Wiklund I, Hedner E, Wygant G, et al. A new instrument for measuring anticoagulation-related quality of life: development and preliminary validation. Health and quality of life outcomes. 2004;2:22-.

26. Norhayati Mohd Noor, Aziz AA. Validity and reliability of the Malay version of 12 -item short form health survey among postpartum mothers. Malays J Public Health Med. 2014;14(2):56-66.

27. Laila Mahmoud Matalqah, Radaideh K. Health-related quality of life among atrial fibrillation patients undergoing anticoagulation therapy. Epidemiol Biostat Public Health. 2018;15(1):e12763-1.

28. Ansell J, Hollowell J, Pengo V, Martinez-Brotons F, Caro J, Drouet L. Descriptive analysis of the process and quality of oral anticoagulation management in real-life practice in patients with chronic non-valvular atrial fibrillation: the international study of anticoagulation management (ISAM). J Thromb Thrombolysis. 2007;23(2):83-91. 
29. Chan PH, Li WH, Hai JJ, Chan EW, Wong IC, Tse HF, et al. Time in therapeutic range and percentage of international normalized ratio in the therapeutic range as a measure of quality of anticoagulation control in patients with atrial fibrillation. Can J Cardiol. 2016;32(10):1247.e23-8.

30. Oh S, Kim J-S, Oh Y-S, Shin D-G, Pak H-N, Hwang G-S, et al. Quality of anticoagulation and treatment satisfaction in patients with non-valvular atrial fibrillation treated with Vitamin K antagonist: result from the Korean atrial fibrillation investigation II. J Korean Med Sci. 2018;33(49):e323-e.

31. Lam MPS, Cheung BMY. The pharmacogenetics of the response to warfarin in Chinese. Br J Clin Pharmacol. 2012;73(3):340-7.

32. van Walraven $C$, Jennings A, Oake N, Fergusson D, Forster AJ. Effect of study setting on anticoagulation control: a systematic review and metaregression. Chest. 2006;129(5):1155-66.

33. Azman AB, Sararaks S, Rugayah B, Low LL, Azian AA, Geeta S, et al. Quality of life of the Malaysian general population: results from a postal survey using the SF-36. Med J Malaysia. 2003;58(5):694-711.

34. Ng DL-C, Gan G-G, Chai C-S, Chee K-H, Tan K-L, Tan S-B, et al. Comparing quality of life and treatment satisfaction between patients on warfarin and direct oral anticoagulants: a cross-sectional study. Patient Preference Adherence. 2019:13:1363-73.

35. Benzimra M, Bonnamour B, Duracinsky M, Lalanne C, Aubert JP, Chassany $\mathrm{O}$, et al. Real-life experience of quality of life, treatment satisfaction, and adherence in patients receiving oral anticoagulants for atrial fibrillation. Patient Prefer Adherence. 2018;12:79-87.

36. Contreras Muruaga MDM, Vivancos J, Reig G, Gonzalez A, Cardona P, Ramirez-Moreno JM, et al. Satisfaction, quality of life and perception of patients regarding burdens and benefits of vitamin K antagonists compared with direct oral anticoagulants in patients with nonvalvular atrial fibrillation. J Comp Eff Res. 2017;6(4):303-12.
37. Alegret JM, Vinolas X, Arias MA, Martinez-Rubio A, Rebollo P, Rafols C, et al. New oral anticoagulants vs vitamin K antagonists: benefits for health-related quality of life in patients with atrial fibrillation. Int J Med Sci. 2014;11(7):680-4.

38. Balci KG, Balci MM, Canpolat U, Sen F, Akboga MK, Suleymanoglu M, et al. Comparison of health-related quality of life among patients using novel oral anticoagulants or warfarin for non-valvular atrial fibrillation. Anatol J Cardiol. 2016;16(7):474-81.

39. De Caterina R, Brüggenjürgen $B$, Darius H, Köhler S, Lucerna M, Pecen $L$, et al. Quality of life and patient satisfaction in patients with atrial fibrillation on stable vitamin $\mathrm{K}$ antagonist treatment or switched to a non-vitamin K antagonist oral anticoagulant during a 1-year follow-up: A PREFER in AF Registry substudy. Arch Cardiovasc Dis. 2018;111(2):74-84.

40. Perino AC, Shrader P, Turakhia MP, Ansell JE, Gersh BJ, Fonarow GC, et al. Comparison of patient-reported care satisfaction, quality of warfarin therapy, and outcomes of atrial fibrillation: findings from the ORBIT-AF registry. J Am Heart Assoc. 2019;8(9):e011205.

41. Aidit S, Soh YC, Yap CS, Khan TM, Neoh CF, Shaharuddin S, et al. Effect of standardized Warfarin treatment protocol on anticoagulant effect: comparison of a Warfarin medication therapy adherence clinic with usual medical care. Front Pharmacol. 2017;8:637-.

\section{Publisher's Note}

Springer Nature remains neutral with regard to jurisdictional claims in published maps and institutional affiliations.
Ready to submit your research? Choose BMC and benefit from:

- fast, convenient online submission

- thorough peer review by experienced researchers in your field

- rapid publication on acceptance

- support for research data, including large and complex data types

- gold Open Access which fosters wider collaboration and increased citations

- maximum visibility for your research: over $100 \mathrm{M}$ website views per year

At BMC, research is always in progress.

Learn more biomedcentral.com/submissions 